\title{
The Rhythm of the Blues
}

\section{ELLEN LEIBENLUFT, M.D.}

For decades, researchers have considered the hypothesis that dysfunction in circadian rhythms and the sleep-wake cycle, rather than simply being symptoms of mood disorders, play a causal role in their pathophysiology. Three studies in this month's Journal continue that line of research. First, Frank et al. (1) reported that patients with bipolar disorder who receive acute treatment with interpersonal and social rhythm therapy show faster improvement in occupational function than do those assigned to intensive clinical management. Second, in a 2-year study of 351 adults ages 60 or older, Cho et al. (2) reported that sleep disturbance predicts the onset of depression, even after the effect of other depressive symptoms is controlled. Finally, Parry et al. (3) reported that levels of the circadian marker melatonin are significantly higher in depressed pregnant women than in euthymic pregnant women, but lower in depressed postpartum women than in euthymic postpartum women.

Basic research relevant to circadian rhythm regulation and the sleep-wake cycle has been advancing at a remarkable pace. Indeed, one could argue that, among physiologic systems regulating complex behavior, the circadian timekeeping system is the first whose molecular genetic underpinnings have, to a considerable extent, been elucidated. Elegant studies have identified how the protein products of such mammalian genes as Clock, $m P e r$, and Cry, expressed in the suprachiasmatic nuclei of the hypothalamus, interact to maintain a rhythm with a "circa-dian" period of about 24 hours (4). The integration of these oscillations, along with the synchronizing effects of light, controls the secretion of melatonin, which occurs primarily during the dark phase of the circadian cycle. With regard to the sleep-wake cycle, the genetic mechanisms and neural circuitry regulating it are complex (including brainstem, hypothalamus, thalamus, and cortex), but sleep research is also making important strides, including identifying the role that sleep plays in memory consolidation, and the regulatory impact of circadian genes on sleep $(5,6)$. In considering a possible etiologic role for circadian and/or sleep-wake dysfunction in mood disorders, it is useful to remember that these two systems, while tightly linked, are dissociable. Specifically, the sleep-wake cycle is regulated not only by oscillatory clock mechanisms, but also by homeostatic mechanisms, so that time elapsed since the last sleep period is an important determinant of sleep propensity (5). Finally, it is important to note that current research on the basic mechanisms mediating circadian rhythms and the sleep-wake cycle is occurring on a backdrop of increased interest in cellular rhythms generally, with data indicating that one physiologic mechanism underpinning the formation of neuronal networks is the synchronization of oscillations at specific frequencies. Using magnetoencephalography, the clinical relevance of this mechanism is being studied in psychiatric illness (e.g., reference 7).

"In the end, the most compelling reason to study sleep-wake and circadian dysfunction in mood disorders is because such studies have the potential to suggest novel therapeutic approaches."

Address correspondence and reprint requests to Dr. Leibenluft, Section on Bipolar Spectrum Disorders, Emotion and Development Branch, Mood and Anxiety Program, NIMH, NIH, HHS, Bldg. 15K, Rm. 203, MSC-2670, Bethesda MD 20892-2670..

The author reports no competing interests. 
Researchers considering the role that circadian rhythms, the sleep-wake cycle, and the interaction between the two may play in the pathophysiology of mood disorders face significant challenges in dissociating cause from effect. Sleep disturbance is a symptom of both mania and depression, but this does not in and of itself demonstrate a causal role for such dysfunction; for this, experimental designs are key. Abundant literature documents that sleep deprivation, prescribed within the context of a controlled study, can have antidepressant effects and, in susceptible patients, precipitate mania (8), demonstrating conclusively that sleep-wake disruption can play a causal role in mood disorders. In the case of circadian rhythms, the data are less extensive, but interventional data in depression suggest the utility of manipulating the relative timing of the sleep-wake cycle and endogenous circadian rhythms $(9,10)$ as well as the possible efficacy of agomelatine, a melatonergic agonist and serotonin $5-\mathrm{HT}_{2 \mathrm{C}}$ antagonist (11). In addition, recent data support possible associations between Clock gene polymorphisms and the symptoms and course of bipolar depression (12).

In this month's Journal, both Frank et al. (1) and Cho et al. (2) tackle the "cause versus effect" issue head-on. Frank et al. (1) is an extension of this group's pioneering work using Interpersonal and social rhythm therapy, an intervention designed to target interpersonal dysfunction and unstable social rhythms, to treat bipolar disorder. Such work has important clinical implications and is also pathophysiologically informative; a previously reported successful clinical trial of Interpersonal and social rhythm therapy with a randomized, controlled design is, in essence, an experiment demonstrating that stabilizing social rhythms, in combination with interpersonal psychotherapy, can increase time to recurrence of an affective episode in patients with bipolar disorder (13). In this month's Journal, Frank et al. (1) reported a post hoc analysis of that trial demonstrating the impact of Interpersonal and social rhythm therapy, in combination with medication, on occupational function. Work performance is severely impaired in most patients with bipolar disorder, but has received little research attention. Comparing patients randomly assigned to Interpersonal and social rhythm therapy to those receiving the comparison treatment, the authors found no difference in occupational function at the end of the acute phase (approximately 35 weeks) or after 2 years. However, those receiving Interpersonal and social rhythm therapy experienced a more rapid improvement in their occupational function than did the comparison group. Interpersonal and social rhythm therapy is a complex intervention, and future work might disentangle its active ingredient(s) e.g., improved interpersonal relationships, stabilization of social rhythms such as meals, etc., and/or sleep stabilization.

Cho et al. (2) also tackled the question of "cause versus effect" in the association between sleep-wake or circadian dysfunction and mood disturbance. Cho et al. (2) used longitudinal clinical data (rather than an experimental design). Compared to randomly controlled trials, longitudinal clinical designs have the disadvantage of being unable to demonstrate causality definitively, but the advantage of larger and more representative samples followed for longer periods. Cho et al. (2) tested whether sleep disturbance predicts depression recurrence in a sample of subjects $\geq 60$ years old living in the community. The subjects were stratified as to whether they had a prior history of depression. Strengths of the study include the focus on older, noninstitutionalized adults; the inclusion of four observations over 2 years; and the fact that the subjects with a prior history of depression had minimal symptoms at baseline. The study's clinical importance is clear: $46 \%$ of the 145 subjects with a history of depression reported sleep disturbance, and $37 \%$ reported using sleep medication within the past month. Even among the 206 subjects with no depressive history, the corresponding numbers were high (18\% and 19\%, respectively). Among those with a depressive history, sleep disturbance predicted the onset of a new depressive episode, even when the analysis controlled for other depressive symptoms and antidepressant medication. As the authors noted, this study is a powerful justification for a clinical trial testing whether aggressive treatment of sleep disturbance can prevent the recurrence of depression in older adults. Hypnotic medications can 
have significant adverse effects in older adults (14) and, in Cho et al. (2), neither hypnotic nor antidepressant medication ameliorated the risk of depressive recurrence. Therefore, nonpharmacological interventions for the treatment of insomnia should receive serious consideration.

Finally, switching focus from the sleep-wake cycle to circadian regulation, Parry et al. (3) compared melatonin levels in pregnant and postpartum women, with and without depression. The study required heroic efforts: pregnant and postpartum women stayed in a dimly lit room for 20 hours, with blood draws every 30 minutes. Compared to controls, melatonin levels were decreased in depressed pregnant women in the morning, and increased in postpartum women throughout the day. These findings complement previous work indicating decreased melatonin levels in women with premenstrual dysphoric disorder and increased levels in those with menopausal depression $(15,16)$. The existence of several interacting regulatory cycles, including those controlling gonadal steroid secretion, melatonin secretion, and the sleep-wake cycle, make the dissociation of cause from effect extremely challenging in this circumstance. For example, the extent to which between-group differences in melatonin secretion are associated with between-group differences in sleep timing or duration is an important question for future research. In any case, the results of this study are other tantalizing hints that circadian rhythm dysfunction may play a role in the pathophysiology of depression, especially when symptoms occur in the context of a female reproductive transition.

In the end, the most compelling reason to study sleep-wake and circadian dysfunction in mood disorders is because such studies have the potential to suggest novel therapeutic approaches, both pharmacological and nonpharmacological. In this month's Journal, this point is demonstrated directly by Frank et al. (1) and Cho et al. (2), with the work of Parry et al. (3) suggesting possible future targets for interventional work. Rapid progress is being made in understanding the basic mechanisms mediating the circadian system, the sleep-wake cycle, and neuronal oscillations. Because of this, and the therapeutic potential in this area, questions concerning the possible impact that dysfunction in these rhythms may play in the pathophysiology of the "blues" provides one of the best opportunities in psychiatry today for translational, bench-to-bedside research.

\section{References}

1. Frank E, Soreca I, Swartz HA, Fagiolini AM, Mallinger AG, Thase ME, Grochocinski VJ, Houck PR, Kupfer DJ. The role of interpersonal and social rhythm therapy in improving occupational functioning in patients with bipolar I disorder. Am J Psychiatry 2008;165:1559-1565. [PubMed: 18829872]

2. Cho HJ, Lavretsky H, Olmstead R, Levin MJ, Oxman MN, Irwin MR. Sleep disturbance and depression recurrence in community-dwelling older adults: a prospective study. Am J Psychiatry 2008;165:15431550. [PubMed: 18765482]

3. Parry BL, Meliska CJ, Sorenson DL, Lopez AM, Martinez LF, Nowakowski S, Elliott JA, Hauger RL, Kripke DF. Plasma Melatonin Circadian Rhythm Disturbances During Pregnancy and Postpartum in Depressed Women and Women With Personal or Family Histories of Depression. Am J Psychiatry 2008;165:1551-1558. [PubMed: 18829869]

4. Turek FW. From circadian rhythms to clock genes in depression. Int Clin Psychopharmacology 2007;22(suppl 2):S1-S8.

5. Fuller PM, Gooley JJ, Saper CB. Neurobiology of the sleep-wake cycle: sleep architecture, circadian regulation, and regulatory feedback. J Biol Rhythms 2006;21:482-493. [PubMed: 17107938]

6. Marshall L, Born J. The contribution of sleep to hippocampus-dependent memory consolidation. Trends Cogn Sci 2007;11:442-450. [PubMed: 17905642]

7. Wilson TW, Rojas DC, Reite ML, Teale PD, Rogers SJ. Children and adolescents with autism exhibit reduced MEG steady-state gamma responses. Biol Psychiatry 2007;62:192-197. [PubMed: 16950225]

8. Wirz-Justice A, Van den Hoofdakker RH. Sleep deprivation in depression: what do we know, where do we go? Biol Psychiatry 1999;46:445-453. [PubMed: 10459393] 
9. Wehr TA, Wirz-Justice A, Goodwin FK, Duncan W, Gillin JC. Phase advance of the circadian sleepwake cycle as an antidepressant. Science 1979;206:710-713. [PubMed: 227056]

10. Benedetti F, Barbini B, Colombo C, Smeraldi E. Chronotherapeutics in a psychiatric ward. Sleep Med Rev 2007;11:509-522. [PubMed: 17689120]

11. Eser D, Baghai TC, Moller HJ. Evidence of agomelatine's antidepressant efficacy: the key points. Int Clin Psychopharmacology 2007;22(suppl 2):S15-S19.

12. Benedetti F, Dallaspezia S, Fulgosi MC, Lorenzi C, Serretti A, Barbini B, Colombo C, Smeraldi E. Actimetric evidence that clock 3111 . T/C SNP influences sleep and activity patterns in patients affected by bipolar depression. Am J Med Genet B Neuropsychiatr Genet 2007;144B:631-635. [PubMed: 17221848]

13. Frank E, Kupfer DJ, Thase ME, Mallinger AG, Swartz HA, Fagiolini AM, Grochocinski V, Houck P, Scott J, Thompson W, Monk T. Two-year outcomes for interpersonal and social rhythm therapy in individuals with bipolar I disorder. Arch Gen Psychiatry 2005;62:996-1004. [PubMed: 16143731]

14. Glass J, Lanctot KL, Herrmann N, Sproule BA, Busto UE. Sedative hypnotics in older people with insomnia: meta-analysis of risks and benefits. BMJ 2005;331:1169-1176. [PubMed: 16284208]

15. Parry BL, Berga SL, Kripke DF, Klauber MR, Laughlin GA, Yen SS, Gillin JC. Altered waveform of plasma nocturnal melatonin secretion in premenstrual depression. Arch Gen Psychiatry 1990;47:1139-1146. [PubMed: 2244799]

16. Parry BL, Meliska CJ, Sorenson DL, López AM, Martínez LF, Nowakowski S, Hauger RL, Elliott JA. Increased melatonin and delayed offset in menopausal depression: role of years past menopause, follicle-stimulating hormone, sleep end time, and body mass index. J Clin Endocrinol Metab 2008;93:54-60. [PubMed: 18042653] 